\title{
Location-dependent Performance of Cooperative Networks with Power Allocation
}

\author{
Tobias Renk and Friedrich K. Jondral \\ Institut für Nachrichtentechnik \\ Universität Karlsruhe (TH) \\ Kaiserstr. 12, 76128 Karlsruhe \\ Email: $\{$ renk,fj\}@int.uni-karlsruhe.de
}

\begin{abstract}
In this paper we investigate a cooperative mobile network with respect to error performance. Power allocation is applied between source and relay. The relay cooperates after having received a request from the destination which decides upon successful or non-successful transmission based on a threshold criterion. We show that with respect to the relay's location a suitable power allocation technique can increase performance enormously. Our results reveal that a practical implementation strategy for cooperative networks first proposed in [1] can easily be adopted to cognitive networks where a relay senses its environment for feedback signals from the destination. Depending on the applied services, however, it may be beneficial to always cooperate, since this creates spatial diversity at the destination.
\end{abstract}

\section{INTRODUCTION}

Recently a new class of transmission called cooperative communications has been introduced. The basic idea behind cooperative communications is that the signal sent from a source to a destination will be overheard by neighboring nodes, so-called relays. These nodes then retransmit the source signal to the destination after prior signal processing. The degree of signal processing depends on the intelligence of the relays. Mostly, three relaying strategies are considered in literature. Amplify-and-forward, where the relays simply amplify the received signal, decode-and-forward, where the relays retransmit a refreshed version of the source signal, and compress-and-forward (sometimes also called quantize-andforward or estimate-and-forward), where the relays retransmit a quantized version of the source signal. Source and relays thus form a virtual multiple-antenna transmitter that creates spatial diversity at the destination.

The conservative way to transmit data is not sufficient anymore for the needs of future multi-media applications due to an increasing number of users and the existence of more resource demanding services. These demands require higher data rates compared to single-hop transmission with one antenna at each node. An alternative way to solve these demands are multiple-input multiple-output (MIMO) systems [2], [3]. At the source and/or at the destination multiple antennas can achieve diversity. This diversity is generated by transmitting a signal from different locations over uncorrelated channels which results in different faded versions of the signal at the destination. In spite of the advantages of increasing link robustness as well as link throughput, MIMO is not suitable for any transmission scenario. Due to size, cost, and/or hardware limitations, mobile nodes can only be equipped with a determined number of antennas. In order to have the benefits of MIMO systems in spite of its limitations, cooperative communications has been introduced. The idea of cooperation (relaying) goes back to van der Meulen [4]. A rigorous information theoretical analysis has been exposed by Cover and El Gamal in 1979 [5]. It was not until 2003 when the interest in cooperation began to rise again [6], [7]. The main reason for this might be limitations in hardware and chip design which made cooperation impossible. In [8] the idea of a self-aware radio named cognitive radio was introduced. An approach to combine the ideas of cooperation and cognition has been made in [9]. The authors give a detailed analysis of the concept of cognitive wireless networks.

The paper is organized as follows. In Section II the system model is described. Section III deals with the evaluation of the system performance. After a general system description, we give a detailed analysis of power allocation fraction, decoding probability at the relay, and the probability of decoding error at the destination. In Section IV the simulation environment is presented and finally Section V concludes the paper.

\section{System Model}

We consider a mobile cooperative network consisting of one source $S$, one relay $R$, and one destination $D$ in this paper (see Fig. 1). Channel gains $\mathbf{h}=\left(h_{\mathrm{sd}}, h_{\mathrm{sr}}, h_{\mathrm{rd}}\right)$ are modeled as independent and circular-symmetric Gaussian random variables with zero means and variances $\sigma_{\mathrm{sd}}^{2}, \sigma_{\mathrm{sr}}^{2}$, and $\sigma_{\mathrm{rd}}^{2}$. The magnitudes then follow a Rayleigh distribution and $|\mathbf{h}|^{2}=$ $\left(\left|h_{\mathrm{sd}}\right|^{2},\left|h_{\mathrm{sr}}\right|^{2},\left|h_{\mathrm{rd}}\right|^{2}\right)$ are exponentially distributed with mean values $\sigma_{\text {sd }}^{2}, \sigma_{\text {sr }}^{2}$, and $\sigma_{\text {rd }}^{2}$ and uniformly distributed phases over $[0,2 \pi)$. A block-fading model is assumed, which means that magnitudes and phases do not change during symbol transmission. Additive white Gaussian noise (AWGN) is added on each channel. Noise follows a complex Gaussian distribution with zero mean and one-sided power spectral density $N_{0}$. The relay performs decode-and-forward and operates in the half-duplex mode, a practical constraint that does not allow reception and transmission of information simultaneously. We assume a power allocation strategy $\mathbf{P}=\left(P_{\mathrm{s}}, P_{\mathrm{r}}\right)=\left(p P_{\text {tot }}, \bar{p} P_{\text {tot }}\right)$, where $p$ is the power allocation fraction and $\bar{p}=1-p$. $P_{\text {tot }}$ is the total transmit power. We furthermore use a common 


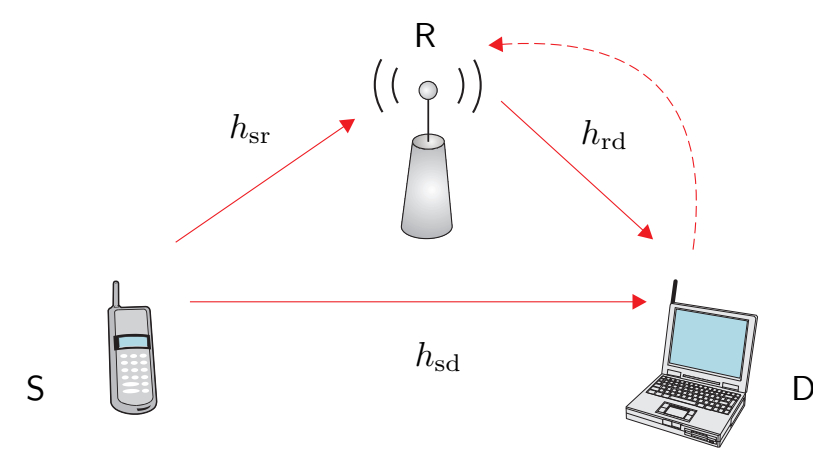

Fig. 1. Network consisting of source $S$, relay R, and destination D. Channel gains are represented by $\mathbf{h}=\left(h_{\mathrm{sd}}, h_{\mathrm{sr}}, h_{\mathrm{rd}}\right)$. Dashed line indicates request for cooperation from the destination.

path loss model to describe fading effects on channels, where $\sigma_{\mathrm{sd}}^{2}=1, \sigma_{\mathrm{sr}}^{2}=d_{\mathrm{sr}}^{-\alpha}$, and $\sigma_{\mathrm{rd}}^{2}=\left(1-d_{\mathrm{sr}}\right)^{-\alpha}$ and $\alpha$ represents the path loss factor. It is between 2.7 and 3.5 for urban cellular radio environments [10] and has been set to 3 for our simulations. Signal-to-noise ratio (SNR) is defined as

$$
\mathrm{SNR}=\frac{P_{\mathrm{tot}}}{N_{0} B}=\frac{E_{b}}{N_{0} B T_{b}}
$$

where $B$ stands for bandwidth, $E_{b}$ denotes bit energy, and $T_{b}$ is bit duration. The receive signal at the destination after direct transmission from the source is described by

$$
y_{\mathrm{sd}}=h_{\mathrm{sd}} x+n_{\mathrm{sd}} .
$$

The time index has been skipped for reasons of presentation and since it is clear from the context. For relay transmission, we have at the destination

$$
y_{\mathrm{rd}}=h_{\mathrm{rd}} \hat{x}+n_{\mathrm{rd}},
$$

where $\hat{x}$ denotes an estimated version of the original source signal, as the relay performs decode-and-forward. Both signals are combined at the destination in terms of maximum ratio combining [11].

\section{Performance Evaluation}

\section{A. System Description}

We investigate a mobile cooperative network, where cooperation takes place if the bit error rate at the destination does not fulfill any predefined requirements. The destination then broadcasts a request (1-bit feedback) to express that cooperation is needed. If no cooperation is needed, the destination remains quiet and all other nodes in the network recognize that direct transmission has been sufficient. This saves energy and time resources in the network and the source can continue by sending the next data frame. Advantage of this structure is the more efficient use of degrees of freedom of the channel, since the relay only cooperates in the mobile environment if really necessary. We assume that the request link from destination to relay is error-free. However, the relay can only cooperate if it was able to decode the source signal reliably. This drawback can be overcome if amplify-and-forward, where the relay

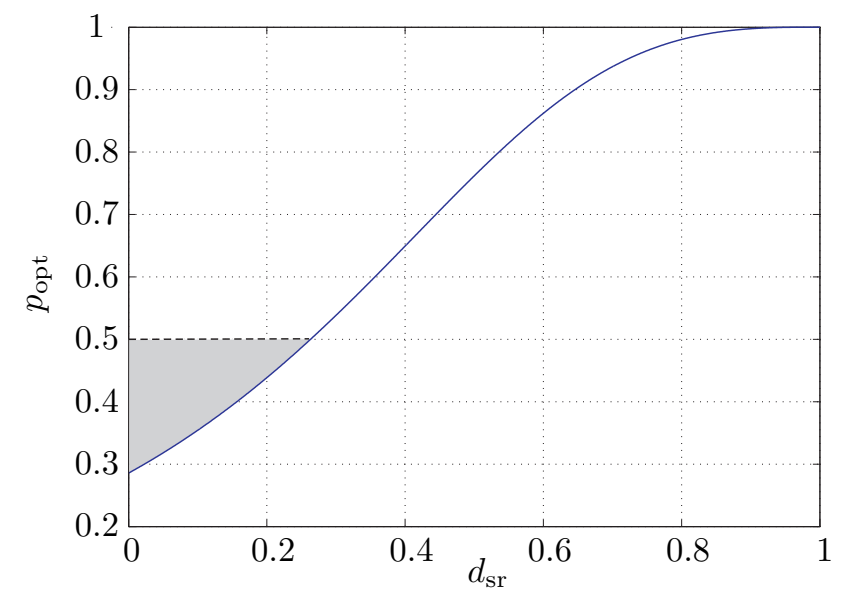

Fig. 2. Optimal power allocation fraction vs. relay location. Grey area indicates interval in which optimal allocation differs from the proposition in [1].

simply amplifies its received signal without prior decoding, is used at the relay. However, amplify-and-forward has the disadvantage of noise enhancement. A hybrid approach is also thinkable. Here, the relay uses decode-and-forward whenever possible. Once it is not able to decode the source signal, it internally switches to amplify-and-forward [12]. After having requested for cooperation, the destination listens to the relay transmission and combines it with the previously received source information applying maximum ratio combining, i.e., the individual SNRs of both transmissions add. This increase in SNR finally yields a lower bit error rate.

\section{B. Power Allocation Fraction}

Power allocation between source and relay in cooperative networks is a proper means to optimize network performance. Mostly, power allocation is investigated with respect to maximizing capacity or, equivalently, minimizing outage probability [13], [14]. In [15] an expression is derived by means of curve-fitting for minimizing error probability at the destination:

$$
p_{\mathrm{opt}}=\frac{1}{1+2.5\left(1-d_{\mathrm{sr}}\right)^{\alpha}}
$$

The function $p_{\text {opt }}\left(d_{\mathrm{sr}}, \alpha\right)$ is monotonically increasing for increasing $d_{\mathrm{sr}}$. It is illustrated in Fig. 2. We clearly see that $p_{\text {opt }} \in[0.285,1]$.

In [1] the authors determined a practical solution to the power allocation problem answering the question of how much of the total transmit power $P_{\text {tot }}$ should be allocated to the source and how much to the relay. The result was that the optimum ratio of source power $P_{\mathrm{s}}$ to total power $P_{\text {tot }}$ is greater than 0.5 and less than 1 , whereas the optimum ratio of relay power $P_{\mathrm{r}}$ to total power $P_{\text {tot }}$ is greater than 0 but less than 0.5. Accordingly,

$$
\frac{1}{2}<\frac{P_{\mathrm{s}}}{P_{\text {tot }}}<1 \quad \text { and } \quad 0<\frac{P_{\mathrm{r}}}{P_{\text {tot }}}<\frac{1}{2} .
$$


Hence, one should always allocate more power to the source than to the relay. If the source-relay link is worse than the link between source and destination, $P_{\mathrm{s}}$ goes to $P_{\text {tot }}$ and $P_{\mathrm{r}}$ goes to 0 . This means that nearly all of $P_{\text {tot }}$ should be used for source transmission and almost nothing for the relay transmission. On the other hand, if the link from source to relay is much better than that from relay to destination, both $P_{\mathrm{s}}$ as well as $P_{\mathrm{d}}$ go to $P_{\text {tot }} / 2$. Expressed in other words, if the quality of the source-relay link is low, it is difficult for the relay to decode the source signal reliably. Therefore, the role of the relay is less important and it makes more sense to put more power to the source. For a good relay-destination link, the relay may be considered as a copy of the source and power should be distributed equally among them. We see that this is almost always the case for optimal power allocation (cf. Fig. 2). For relay locations greater than approximately $0.27, p_{\text {opt }}$ is greater than 0.5 .

\section{Detection at the Relay}

In this subsection, we derive the probability for detection at the relay. The relay can decode if the rate at which the source transmits is less than the mutual information between source and relay, i.e., $I\left(X_{\mathrm{S}} ; Y_{\mathrm{R}}\right)>R$. Expressed in terms of communications, this corresponds to the event that the received SNR at the relay is higher than the required threshold SNR. Considering the fact that $\left|h_{\mathrm{sr}}\right|^{2}$ is exponentially distributed and applying our path loss model, we have

$$
\begin{aligned}
P_{\text {decode }} & =\operatorname{Pr}\left(\left|h_{\mathrm{sr}}\right|^{2}>\frac{\mathrm{SNR}_{\mathrm{th}}}{p \mathrm{SNR}}\right) \\
& =\exp \left(-\frac{\mathrm{SNR}_{\mathrm{th}} d_{\mathrm{sr}}^{\alpha}}{p \mathrm{SNR}}\right) .
\end{aligned}
$$

Information theoretical tools provide a proper expression of the threshold SNR, which is

$$
\mathrm{SNR}_{\mathrm{th}}=2^{R}-1 \text {. }
$$

In [15] an expression of the optimal threshold SNR for BPSK/QPSK modulation is given by

$$
\mathrm{SNR}_{\mathrm{th}}^{(\mathrm{opt})} \approx 3 \sqrt{\frac{2 p \log _{10}(\mathrm{SNR})}{d_{\mathrm{sr}}^{\alpha}}},
$$

if applied with optimal power allocation fraction $p_{\text {opt }}$. Noteworthy, that we already adopted this equation to our considered transmission environment.

\section{Error Probability at the Destination}

We define two events in order to describe overall transmission in the mobile network. Let $\mathcal{A}=$ \{direct transmission and $\mathcal{B}=\{$ cooperative transmission $\}$. Let $\mathcal{S}_{\text {net }}$ be the network transmission state. Then we have:

$$
\mathcal{S}_{\text {net }}= \begin{cases}\mathcal{A} & \text { if } P_{\mathcal{A}}<P_{e}^{(\mathrm{th})} \\ \mathcal{B} & \text { if } P_{\mathcal{A}} \geq P_{e}^{(\mathrm{th})}\end{cases}
$$

We now have to determine the error probabilities for the two network transmission states $\mathcal{A}$ and $\mathcal{B}$. $\mathcal{A}$ is the trivial case of direct transmission. This error probability is well-known for Rayleigh fading channels and can be looked up in [10], [16], [17], for instance. For BPSK/QPSK we have a bit error rate of

$$
P_{e}=P_{\mathcal{A}}=\frac{1}{2}\left(1-\sqrt{\frac{p \mathrm{SNR}}{1+p \mathrm{SNR}}}\right),
$$

where $p$ SNR is the average SNR on the source-destination link since $d_{\mathrm{sd}}=1$. Error probability for the network transmission state $\mathcal{B}$ is more involved. Now we have to consider if the relay has been able to decode the source signal. If so, spatial diversity is created at the destination. If not, the destination merely receives the source signal. Consequently,

$$
P_{e}=P_{\mathcal{B}}=P_{\text {decode }} P_{e}^{(\text {div })}+\bar{P}_{\text {decode }} P_{\mathcal{A}}
$$

with $\bar{P}_{\text {decode }}=1-P_{\text {decode }}$. The only unknown variable of the above equation is $P_{e}^{\text {(div) }}$. According to [15], it depends on the probability of decoding error at the relay $P_{e}^{(\mathrm{rel})}$ and the probability of decoding error at the destination after combining the source and the relay signal $P_{e}^{(\mathrm{dest})}$. Hence,

$$
P_{e}^{(\mathrm{div})}=P_{e}^{(\mathrm{rel})} P_{e}^{(\mathrm{con})}+\bar{P}_{e}^{(\mathrm{rel})} P_{e}^{(\mathrm{dest})}
$$

where $P_{e}^{(\mathrm{con})} \leq 0.5$ denotes a consecutive error (i.e., an error at the relay is propagated to the destination) and $\bar{P}_{e}^{(\mathrm{rel})}=$ $1-P_{e}^{(\mathrm{rel})} . P_{e}^{(\mathrm{rel})}$ shows a similar structure to (11) by simply replacing $p$ SNR by $p d_{\mathrm{sr}}^{-\alpha} \mathrm{SNR}$. A solution to $P_{e}^{(\text {dest })}$ for BPSK/QPSK is given in [15],

$$
P_{e}^{(\mathrm{dest})}=\frac{1}{2}\left(1-\frac{f(\mathrm{SNR})-g(\mathrm{SNR})}{p \mathrm{SNR}-\bar{p} d_{\mathrm{rd}}^{-\alpha} \mathrm{SNR}}\right),
$$

where

$$
f(\mathrm{SNR})=p \mathrm{SNR} \sqrt{\frac{p \mathrm{SNR}}{1+p \mathrm{SNR}}}
$$

and

$$
g(\mathrm{SNR})=\bar{p} d_{\mathrm{rd}}^{-\alpha} \mathrm{SNR} \sqrt{\frac{\bar{p} d_{\mathrm{rd}}^{-\alpha} \mathrm{SNR}}{1+\bar{p} d_{\mathrm{rd}}^{-\alpha} \mathrm{SNR}}} .
$$

We now have described the error performance of the overall network depending on a bit error rate threshold at the destination. If the bit error rate at the destination is below this threshold, $P_{e}=P_{\mathcal{A}}$. If not, we have $P_{e}=P_{\mathcal{B}}$.

\section{Simulation Results}

The simulation environment is based on the IEEE 802.11 standard. However, significant changes have been made in order to fit the protocol to our network structure. We use a modified version of the distributed coordination function (DCF). DCF is a random access scheme based on carrier sense multiple access with collision avoidance (CSMA/CA). We employ the request-to-send/clear-to-send (RTS/CTS) mechanism. In order to handle the feedback request and the additional transmission by the relay, we introduce a second RTS frame. The packet lengths are similar to those of the standard defined in [18]. In order to manage medium access we introduce a new frame that is sent by the relay to reserve the channel. First, 


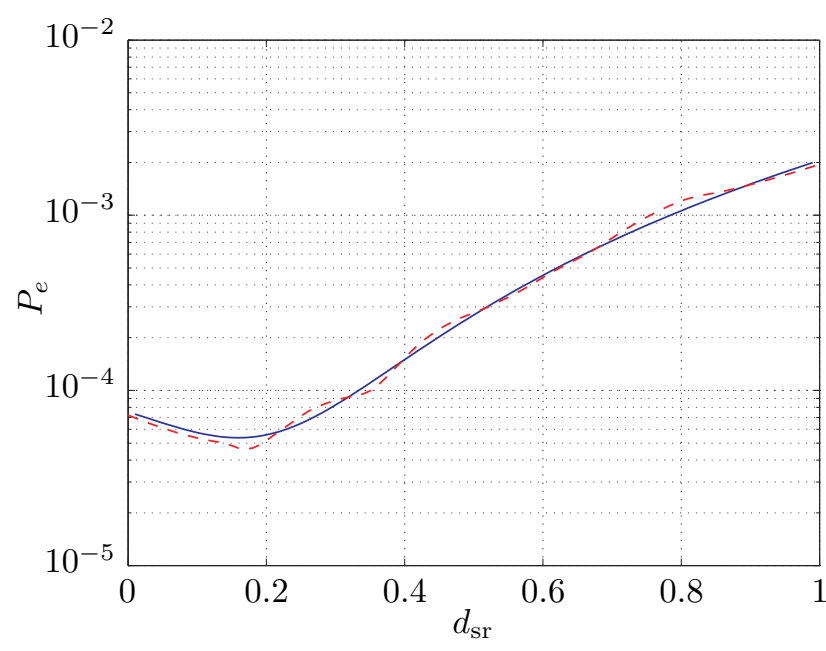

Fig. 3. Bit error rate vs. relay position for $p=0.6$ and $E_{b} / N_{0}=20 \mathrm{~dB}$. Solid lines indicate analytical and dashed lines simulation results, respectively.

the source transmits an RTS and the destination does not reply to that frame immediately, but wait for another RTS frame from the relay (which includes data that indicates that this is the frame from the relay not the source). After reception of this RTS, the destination sends a CTS which includes data that manages medium access for source and relay. When the source message has been sent, the relay stays idle for a short predefined time in order to be able to receive feedback from the destination. If this feedback is positive, i.e., no relay transmission is needed, the destination sends an ACK and the contention phase begins again. If the feedback is negative, i.e., relay transmission is necessary, the relay transmits and after reception of the relay message the destination sends an ACK. In contrast to the aforementioned aspects, we next present results where no feedback request has been applied and the relay has always retransmitted a refreshed version of the source signal.

Fig. 3 illustrates bit error rate at the destination over the relay location. A power allocation fraction of $p=0.6$ has been assigned to the source and the parameter for the simulation was $E_{b} / N_{0}=20 \mathrm{~dB}$. We see that there is an optimal relay location of $d_{\mathrm{sr}}^{(\mathrm{opt})}=0.17$ where the bit error rate has a minimum. Moving the relay closer to the destination leads to a remarkable increase of bit error rate. This result is in line with the fact that decode-and-forward performs better in networks where the relay is placed close to the source [19].

In Fig. 4 bit error rate over power allocation fraction $p$ is shown. The relay has been placed at $d_{\mathrm{sr}}=0.85$ and $E_{b} / N_{0}$ was set to $20 \mathrm{~dB}$. It can easily be seen that the result derived in [1], i.e., putting more power into the source transmission rather than the relay transmission, leads to a better error performance. However, for $p=1$ the bit error rate curve increases highly. This is due to the fact that for $p=1$ all power is allocated to the source and no power to the relay. Hence, we have a direct transmission scheme and the benefit of spatial diversity cannot be exploited any more at the destination.

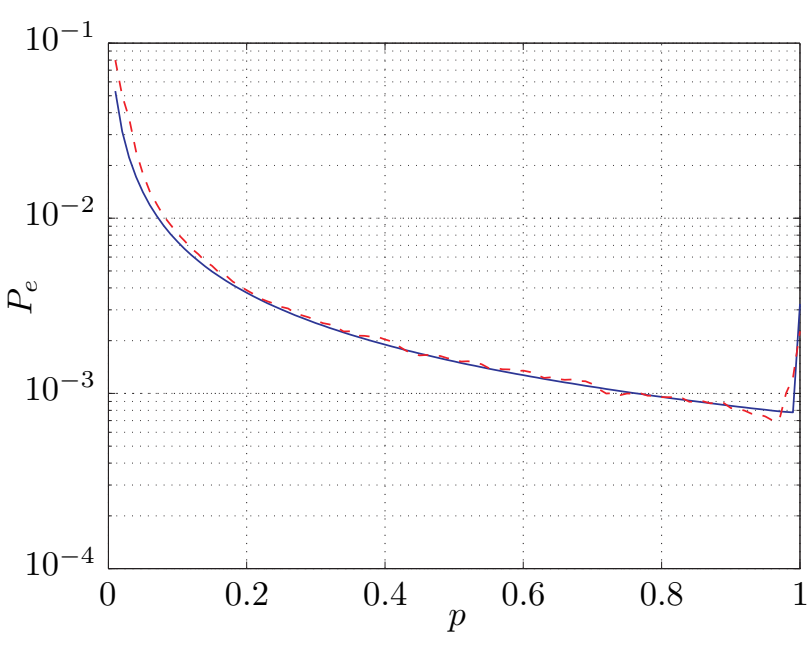

Fig. 4. Bit error rate vs. power fraction for $d_{\mathrm{sr}}=0.85$ and $E_{b} / N_{0}=20 \mathrm{~dB}$. Solid lines indicate analytical and dashed lines simulation results, respectively.

In Fig. 5 we compare direct transmission to cooperative transmission for $p=0.6$ and $d_{\mathrm{sr}}=0.3$. Cooperative transmission always outperforms direct transmission for similar $E_{b} / N_{0}$ values. Especially, we see the increase in diversity created by cooperation (slope of the bit error rate curve) which can be calculated as

$$
d=-\lim _{\mathrm{SNR} \rightarrow \infty} \frac{\log P_{e}(\mathrm{SNR})}{\log \mathrm{SNR}} .
$$

Changing $p$ and $d_{\mathrm{sr}}$ values provides again that allocating more power to the source rather than the relay and placing the relay closer to the source achieves better error performance.

Next, we show the error performance of the investigated network when a feedback request is introduced. We chose the threshold bit error rate to be $P_{e}^{(\mathrm{th})}=10^{-2}$. The relay has been placed on a straight line between source and destination at $d_{\mathrm{sr}}=0.6$. We see in Fig. 6 that up to a power allocation fraction of $p=0.04$ the required bit error rate cannot be fulfilled. In order to get the closest performance to the required threshold, however, cooperative communications is employed. Cooperative transmission continues to $p \approx 0.27$. Then, direct transmission achieves a bit error rate lower than $10^{-2}$. From that point on, the whole network is in the network transmission state $\mathcal{S}_{\text {net }}=\mathcal{A}$.

\section{CONCLUding Remarks And Future Work ITEMS}

In this paper we investigated a cooperative mobile network with respect to error performance. We applied power allocation between source and relay in terms of minimizing error probability. In order to use degrees of freedoms of the channel and network resources more efficiently, cooperative communications only takes place if the relay has received a request from the destination node. A request is sent if a required bit error rate threshold cannot be fulfilled. Our results reveal that a practical implementation strategy for cooperative networks also proposed in [1] is the following: i) Power allocation fraction $p$ should be selected $\geq 0.5$, ii) it 


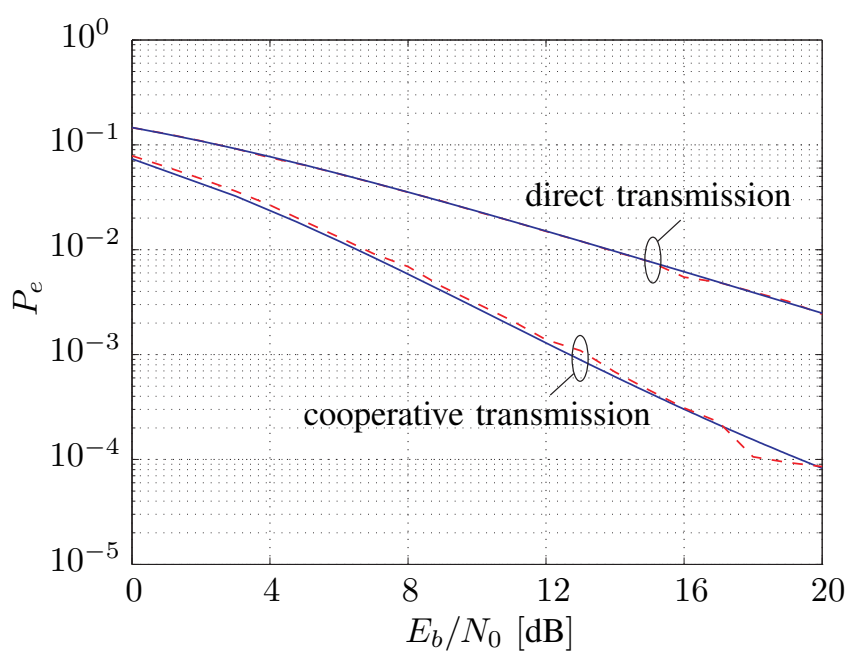

Fig. 5. Bit error rate vs. $E_{b} / N_{0}$ for direct transmission and cooperative transmission with $p=0.6$ and $d_{\mathrm{sr}}=0.3$. Solid lines indicate analytical and dashed lines simulation results, respectively.

is advantageous to place the relay nearby the source if decodeand-forward is applied.

There are several open points that can increase system performance in some point of view. For instance, a hybrid relaying strategy could be employed. This means that the relay always forwards the source signal. Depending on its own decoding ability, however, it selects between amplifyand-forward and decode-and-forward on its own. This clearly can improve performance and there is no need for feedback then. However, the relay often transmits when this is actually not necessary. This leads to a waste of resources, e.g., battery power, which is a critical issue in mobile networks like ad-hoc networks. We have only considered one relay in the network. Several issues arise with the introduction of more relays. In [20] the number of neighbors a wireless node can directly communicate with is investigated. Results reveal the great potential of beamforming which has not been considered in this paper.

\section{ACKNOWLEDGMENT}

The authors would like to thank Samuel Hatorangan for his contributions in building up the simulator.

\section{REFERENCES}

[1] S. Weifeng, A. K. Sadek, and K. J. Ray Liu, "Cooperative communication protocols in wireless networks: Performance analysis and optimum power allocation," Wireless Personal Communications, vol. 44, no. 2, pp. 181-217, 2008.

[2] G. Foschini and M. J. Gans, "On limits of wireless communications in a fading environment when using multiple antennas," Wireless Personal Communications, Kluwer Academic Publishers, vol. 6, pp. 311-335, 1998.

[3] İ. E. Telatar, "Capacity of multi-antenna gaussian channels," European Transactions on Telecommunications, vol. 10, pp. 585-595, November/December 1999.

[4] E. van der Meulen, "Transmission of information in a t-terminal discrete memoryless channel," Department of Statistics, University of California, Berkeley, CA, Technical Report, 1968.

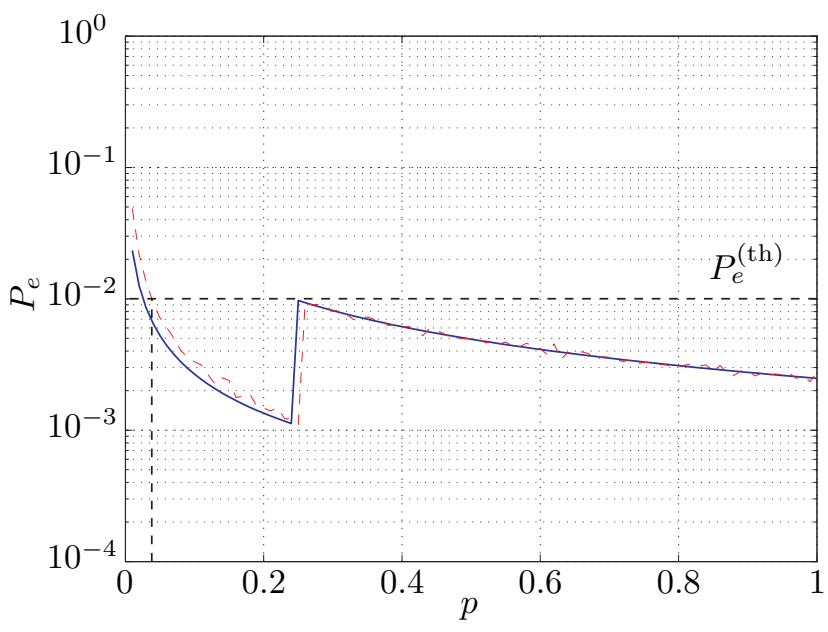

Fig. 6. Bit error rate vs. power fraction for $d_{\mathrm{sr}}=0.6$ and $P_{e}^{(\mathrm{th})}=10^{-2}$. Solid lines indicate analytical and dashed lines simulation results, respectively.

[5] T. Cover and A. El Gamal, "Capacity theorems for the relay channel," IEEE Transactions on Information Theory, vol. 25, no. 5, pp. 572-584, September 1979.

[6] A. Sendonaris, E. Erkip, and B. Aazhang, "User cooperation diversity - part I: System description," IEEE Transactions on Communications, vol. 51, pp. 1927-1938, 2003.

[7] — , "User cooperation diversity - part II: Implementation aspects and performance analysis," IEEE Transactions on Communications, vol. 51, pp. 1939-1948, 2003.

[8] J. Mitola, "Cognitive radio: An integrated agent architecture for software defined radio," Ph.D. dissertation, Royal Institute of Technology (KTH) Stockholm, Sweden, May 2000.

[9] F. H. P. Fitzek and M. Katz (Eds.), Cognitive Wireless Networks: Concepts, Methodologies and Visions Inspiring the Age of Enlightenment of Wireless Communications. Springer, 2007.

[10] T. Rappaport, Wireless Communications: Principles \& Practice. New Jersey: Prentice Hall, 1996.

[11] D. Brennan, "Linear diversity combining techniques," Proceedings of the IRE, vol. 47, no. 1, pp. 1075-1102, 1959.

[12] M. Souryal and B. Yojcic, "Performance of amplify-and-forward and decode-and-forward relaying in rayleigh fading with turbo codes," IEEE International Conference on Acoustics, Speech, and Signal Processing (ICASSP), Toulouse, France, May 2006.

[13] R. Cruz and A. Santhanam, "Optimal routing, link scheduling and power control in multi-hop wireless networks," IEEE Infocomm, San Francisco, USA, 2003.

[14] A. Høst-Madsen and J. Zhang, "Capacity bounds and power allocation for wireless relay channels," IEEE Transactions on Information Theory, vol. 51, no. 6, pp. 2020-2040, June 2005.

[15] P. Herhold, E. Zimmermann, and G. Fettweis, "A simple cooperative extension to wireless relaying," Int. Zurich Seminar on Communications (IZS), February 2004.

[16] J. Proakis, Digital Communications, 4th ed. New York: McGraw-Hill, 2001.

[17] S. Stein, "Fading channel issues in system engineering," IEEE Journal on Selected Areas in Communications, vol. 5, no. 2, pp. 68-89, February 1987.

[18] IEEE 802.11-2007, "Standard for LAN/MAN - Specific requirements Part 11: Wireless LAN Medium Access Control (MAC)and Physical Layer (PHY) Specifications," 2007.

[19] G. Kramer, M. Gastpar, and P. Gupta, "Cooperative strategies and capacity theorems for relay networks," IEEE Transactions on Information Theory, vol. 51, no. 9, pp. 3037-3063, September 2005.

[20] C. Hartmann, M. Kiese, and R. Vilzmann, "Optimizing the node degree in wireless multihop networks with single-lobe beamforming," The 9th INFORMS Telecommunications Conference, College Park, Maryland, USA, March 2008. 